\title{
Depth-Resolved Profile of the Magnetic Field beneath the Surface of a Superconductor with a Few nm Resolution
}

\author{
T. J. Jackson, ${ }^{1}$ T. M. Riseman, ${ }^{1}$ E. M. Forgan, ${ }^{1}$ H. Glückler, ${ }^{2}$ T. Prokscha, ${ }^{2}$ E. Morenzoni, ${ }^{2}$ M. Pleines,${ }^{2,3}$ \\ Ch. Niedermayer, ${ }^{3}$ G. Schatz, ${ }^{3}$ H. Luetkens, ${ }^{2,4}$ and J. Litterst ${ }^{4}$ \\ ${ }^{1}$ School of Physics and Astronomy, University of Birmingham, Birmingham B15 2TT, United Kingdom \\ ${ }^{2}$ Labor für Myonspinspektroskopie, Paul Scherrer Institut, CH-5232 Villigen PSI, Switzerland \\ ${ }^{3}$ Universität Konstanz, Fakultät für Physik, D-78434 Konstanz, Germany \\ ${ }^{4}$ Technische Universität Braunschweig, D-38106 Braunschweig, Germany
}

(Received 7 February 2000)

\begin{abstract}
The variation of a magnetic field as a function of depth beneath the surface of an $\mathrm{YBa}_{2} \mathrm{Cu}_{3} \mathrm{O}_{7-\delta}$ thin film in the Meissner state has been measured using low energy muons. The depth of implantation was varied from 20-150 nm by tuning the energy of the implanted muons from 3-30 keV. These are direct measurements of the penetration of a magnetic field beneath a superconducting surface which illustrate the power of low energy muons for near surface studies in superconductivity and magnetism.
\end{abstract}

PACS numbers: 74.25.Ha, 74.76.Bz, 76.75.+i

The determination of the value of a magnetic field as a function of depth below a surface is a nontrivial problem because a microscopic probe is required to sample the local fields in this region. In this Letter we report the use of low energy muons [1] to profile directly the magnetic field beneath the surface of a superconductor in the Meissner state, with a depth resolution of a few nanometers. A magnetic field was applied parallel to the surface of a $c$-axis oriented superconducting $\mathrm{YBa}_{2} \mathrm{Cu}_{3} \mathrm{O}_{7-\delta}$ (YBCO) thin film after zero field cooling the film to $20 \mathrm{~K}$. In this geometry, currents flowing in the $a b$ planes determine the profile along the crystal $c$ axis of the magnetic field inside the film. Muons were implanted at depths from $20-150 \mathrm{~nm}$ by varying the energy of the incident muons from 3-30 keV. This allowed us to measure values of magnetic field as a function of depth. The large value of $H_{c 1}$ at low temperatures, surface barriers, and flux pinning all prevent flux line entry [2], so in these measurements we are observing Meissner screening. We have previously reported the use of low energy muons to monitor the spatial evolution of the magnetic field distribution in the mixed state as flux lines emerge through the surface of an YBCO thin film [3]. In those measurements the magnetic field was applied perpendicular to the surface of the film, and the sample was field cooled through the transition temperature $T_{c}$ to obtain the mixed state.

In the Meissner state, diamagnetic shielding currents flowing close to the sample surface screen a static magnetic field from the interior of the body. We may define an average depth of penetration $\langle\lambda\rangle$ of an external field $B_{0}$ by

$$
\langle\lambda\rangle=\frac{1}{B_{0}} \int B(z) d z
$$

which is the quantity given, for example, by microwave measurements of field penetration [4]. However, other techniques such as muon spin rotation ( $\mu \mathrm{SR})$ [5] will give different average quantities which we shall generically denote by $\lambda$.
Measurements of the penetration depth provide information crucial to understanding the details of the superconducting state [6]. The field causes a shift in momentum space of the whole of the Fermi sphere. At finite temperature or in regions where the gap is zero, this changes the occupation of quasiparticle excitation states. The effective number density of superconducting charge carriers $n_{s}$ participating in the screening currents is thus reduced from the total number density of carriers. In the limit where the penetration depth is much larger than the coherence length $\xi_{0}$, and where the mean free path for scattering of the normal electrons $l \gg \xi_{0},\langle\lambda\rangle$ is the familiar London penetration depth $\lambda_{L}$,

$$
\lambda_{L}^{2}(T)=\frac{m_{s}}{(2 e)^{2} \mu_{0} n_{s}(T)},
$$

where $m_{s}$ is the effective mass of the carriers. It is well known that in this London limit a magnetic field applied parallel to the surface of a semi-infinite superconducting slab decays with depth $z$ according to the exponential decay law

$$
B(z)=B_{0} \exp \left(-z / \lambda_{L}\right) .
$$

In this case the average depth of penetration given by Eq. (1) is equal to $\lambda_{L}$. However, whenever the field penetration is nonexponential, any technique which measures $\langle\lambda\rangle$ is not directly giving $\lambda_{L}$ for a material.

If $\lambda_{L} \ll \xi_{0}$ (the nonlocal, Pippard limit) the average depth of penetration of the field is increased. Furthermore, the field penetration becomes nonexponential, and indeed the direction of the field reverses at depths somewhat larger than $\langle\lambda\rangle$ [7]. However, to a first approximation, high temperature superconductors (HTS) are expected to be close to the London limit.

An interesting feature of HTS is that they have $d$-wave gap nodes as revealed, for instance, by microwave measurements [4]. This can lead to nonlinear effects of field on 
penetration depth, associated with a field induced increase in the density of available excitation states located near the gap nodes [8]. A nonlocal effect is associated with the divergence of $\xi_{0}$ at the same nodes, as $\xi_{0}$ varies in inverse proportion to the value of the energy gap [9]. However, ac susceptibility measurements of the dependence of $\langle\lambda\rangle$ on the magnitude and orientation of the applied field with respect to the $a$ and $b$ axes of YBCO [10] have shown a much smaller nonlinear effect than predicted [8]. Recent $\mu$ SR measurements [5] showed a field-induced increase in $\lambda$ and a saturation of $\lambda(\mathrm{T})$ at low temperatures, which were attributed to nonlinear and nonlocal effects. These measurements illustrate how penetration depth measurements address the essential physics of superconductors.

In the mixed state, the penetration depth can be deduced from neutron scattering [11], $\mu \mathrm{SR}$, or from imaging of a single flux line emerging from a surface using electron holography [12] or SQUID microscopy [13]. Alternatively, the field below the surface in the Meissner state can be inferred from the height-dependent force profile above the surface as measured by magnetic force microscopy [14]. But in all of these examples the London law, which leads to Eq. (3), is assumed. For example, in conventional $\mu \mathrm{SR}$ the distribution of internal magnetic fields $P(B)$ deduced from the experimental data is compared with the distribution calculated for the flux line lattice present in the superconducting mixed state. This calculated $P(B)$ is derived by assuming a model of the structure of the flux line based on the London law, modified by flux line core effects [5]. Our previous low energy muon experiments [3] also relied on the use of the London model to fit the data. Spin-polarized neutron reflectivity is sensitive to the field profile $[2,15]$ but requires model fitting of spin-dependent intensities to deduce the value of $\lambda$. Hence, none of these methods directly determine the profile of the field within the superconductor.

A direct measure of the spatial variation of the magnetic field can be obtained with the use of low energy muons. This technique differs in principle and not just in methodology or geometry from the examples given above, in that no assumptions are made concerning the details of the field penetration law. In conventional $\mu \mathrm{SR}, 4 \mathrm{MeV}$ spinpolarized muons are implanted within bulk material and stop over a range of depths of order $0.3 \mathrm{~mm}$ (with a straggle of $0.07 \mathrm{~mm}$ FWHM). The temporal evolution of the polarization of the implanted muons is indicated by the direction of emission of decay positrons. The magnetic field at each muon implantation site causes precession of the muon spin. The positron time spectra collected from an ensemble of muons contain oscillations which reveal the distribution $P(B)$ of local magnetic fields. With low energy $\mu \mathrm{SR}$, similar measurements can be performed, but over much smaller depth ranges. In the low energy $\mu \mathrm{SR}$ spectrometer at the Paul Scherrer Institute [1] the muons are moderated in a condensed van der Waals gas layer to energies $\sim 15 \mathrm{eV}$. These epithermal muons are then extracted from the moderator by applying up to $20 \mathrm{kV}$ to the moderator substrate. The final implantation energy is determined by the extraction potential and by an accelerating or decelerating potential applied to the sample, which is mounted via an electrically insulating sapphire plate on a continuous flow cryostat. The incoming muons are detected by a $10 \mathrm{~nm}$ thick carbon foil placed at an intermediate focus of the beam transport system, upstream of the sample. Secondary electrons are emitted as the muon passes through the foil and are amplified by a microchannel plate to provide a trigger signal [16].

The sample in this experiment was a $50 \mathrm{~mm}$ diameter, $700 \mathrm{~nm}$ thick high quality YBCO film, grown by thermal coevaporation of the constituents onto a single crystal lanthanum aluminate substrate [17]. The transition temperature $T_{c}$ was measured by ac susceptibility to be $87.5 \mathrm{~K}$. The film had the crystal $c$ axis perpendicular to the plane and the twinned $a b$-planes were well aligned with the substrate. The surface roughness of the YBCO film was measured by atomic force microscopy. The deviations of height from the mean had an rms value of $5 \mathrm{~nm}$, which corresponds to a thickness uniformity of better than $1 \%$. The thickness varied on in-plane distance scales that ranged from a fraction of a micron downwards.

The magnetic field of $9.5 \mathrm{mT}$ was parallel to the film surface and perpendicular to the incoming muon momentum, and perpendicular to the spin of the transversely polarized muons. In a typical experimental run, some 80000 muon decays were recorded, each as a function of time after detection of an incoming muon. The distribution $P(B)$ of the values of magnetic field experienced by the implanted muons was derived by maximum entropy Fourier analysis $[18,19]$ of the muon precession. In Fig. 1 we show the resulting magnetic field distributions obtained at $20 \mathrm{~K}$ for various implantation energies. It is immediately clear that

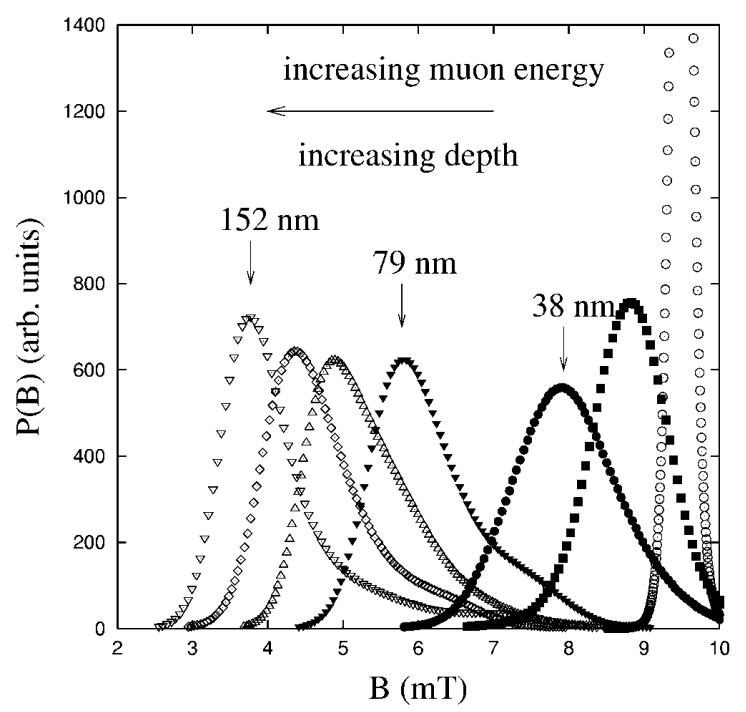

FIG. 1. Field distributions observed in YBCO at $20 \mathrm{~K}$ in the Meissner state with muons implanted at (ם) $3.4 \mathrm{keV}$, (○) $6.9 \mathrm{keV},(\boldsymbol{\nabla}) 15.9 \mathrm{keV},(\triangle) 20.9 \mathrm{keV},(\diamond) 24.9 \mathrm{keV}$, and ( $) 29.4 \mathrm{keV}$. The field distribution shown with open circles was taken in the normal state. 
in the superconducting state the higher the incident muon energy, the lower the average field. The narrow (resolution limited [19]) field distribution at $9.5 \mathrm{mT}$ shown with open circles was taken at $100 \mathrm{~K}$-i.e., in the normal state, and is independent of implantation energy.

Muons of a given energy stop over a certain range of depths, giving the small range of fields in $P(B)$. The muon implantation depth distribution $p(z)$ corresponding to each implantation energy marked in Fig. 1 was calculated using a Monte Carlo code TRIM.SP (transport and range of ions in matter) [20] and is shown in Fig. 2. We have shown elsewhere that this program predicts the implantation profiles accurately [21]. A Gaussian spread of input muon energies of $0.5 \mathrm{keV}$, corresponding to the energy straggling introduced by incoming muon detector [22], is included in the calculations.

To an excellent first approximation, to plot the field $B$ as a function of depth $z$, we simply read off the peak fields in Fig. 1 and plot them versus the peak depths in Fig. 2. This plot allows one to iterate rapidly to the correct relationship between $P(B)$ and $p(z)$ :

$$
P(B)=\frac{p[z(B)]}{d B / d z} .
$$

Equation (4) shows that the value of the field $B_{\text {peak }}$ for the maximum of $P(B)$ is at a depth $z_{\text {peak }}$ where $p(z) /(d B / d z)$ is maximum - rather than where $p(z)$ is maximum; the correction introduced by using Eq. (4) is only of order $1 \mathrm{~nm}$. The resulting values of $B_{\text {peak }}$ versus $z_{\text {peak }}$ after making this small correction are plotted for several sample temperatures in Fig. 3. The theoretical lines are plots of the function:

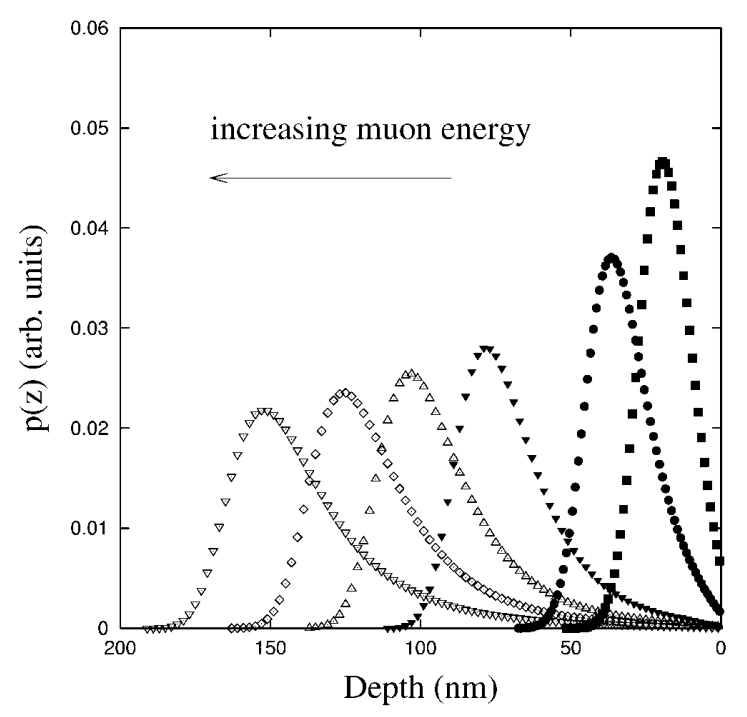

FIG. 2. Low energy muon implantation profiles for muons with the same energies as in Fig. 1, calculated using TRIM.SP. The profiles have been convoluted with a Gaussian width of $5 \mathrm{~nm}$ to represent depth straggling due to surface roughness of the film. The asymmetrical shape of the profile is reflected in the asymmetry of the field distributions in Fig. 1.

$$
B(z)=B_{0} \frac{\left.\cosh \left[(t-z) / \lambda_{L}\right)\right]}{\cosh \left(t / \lambda_{L}\right)} .
$$

This is the form taken by Eq. (3) for a film of thickness $2 t$, with flux penetrating from both surfaces. The value of $z$ in Eq. (5) has been corrected by a small quantity $z_{0}$, corresponding to a "dead layer." This may partly be due to a thin layer that is nonsuperconducting, but arises mainly from the surface roughness of the film, which increases the effective penetration depth in the surface layers. It may be seen that in the surface region where the superconducting planes are not continuous, the effective penetration depth includes the $c$-axis penetration depth, because shielding currents cannot flow continuously in the $a b$ planes alone; hence the field penetrates into the "sides" of any "hills" on the surface. The $c$-axis penetration depth is a factor $\gamma \sim 5-8$ times longer than that due to currents in the basal plane. Thus, if the surface roughness corresponds to a thickness $\delta$ of full-density material, then the muons will have traversed on average a depth $\delta$, but the field will only have been shielded by an amount corresponding to $\delta / \gamma$ of full-density $a b$-plane material. Hence the expected value of $z_{0}=\delta(1-1 / \gamma)$, which with the measured surface roughness will correspond to a few $\mathrm{nm}$. These considerations apply so long as the surface roughness has a scale and height much smaller than the relevant penetration depths, which applies in our case. The small horizontal scale of the surface roughness also has the consequence that local variations in field produced by hills and valleys on the surface rapidly die away with depth. In initial fits

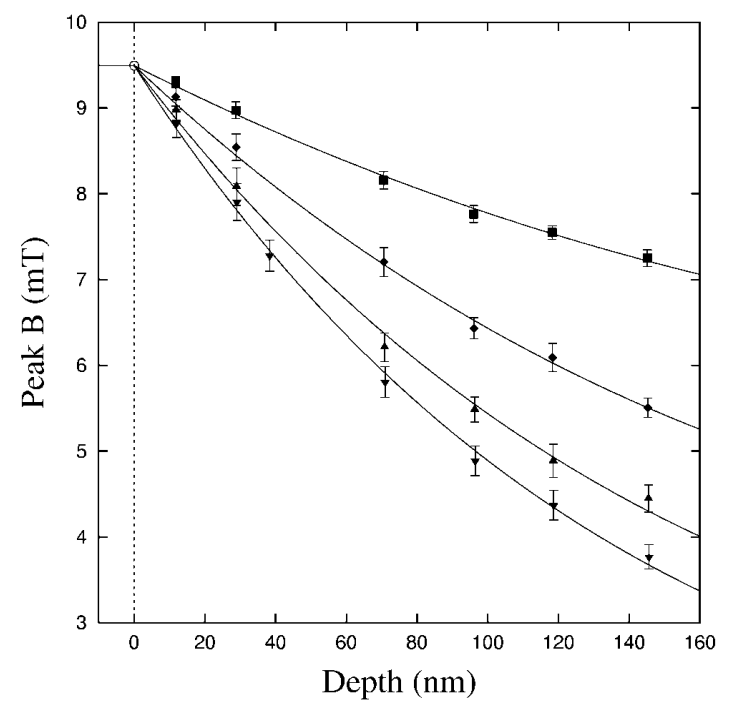

FIG. 3. Values of field versus depth for various values of sample temperature $(\boldsymbol{\nabla}) 20 \mathrm{~K},(\boldsymbol{\Delta}) 50 \mathrm{~K},(\bullet) 70 \mathrm{~K}$, and (a) $80 \mathrm{~K}$. The solid lines represent fits of Eq. (5) to the data with $\lambda_{L}$ as the free parameter. The implantation depth has been corrected by a fixed amount $z_{0}$ mainly to allow for the slight surface roughness of the sample. The thickness $2 t$ was not corrected, because $z_{0}$ is comparable with the error in the thickness and small changes in $2 t$ have a negligible effect on the fit parameters. 


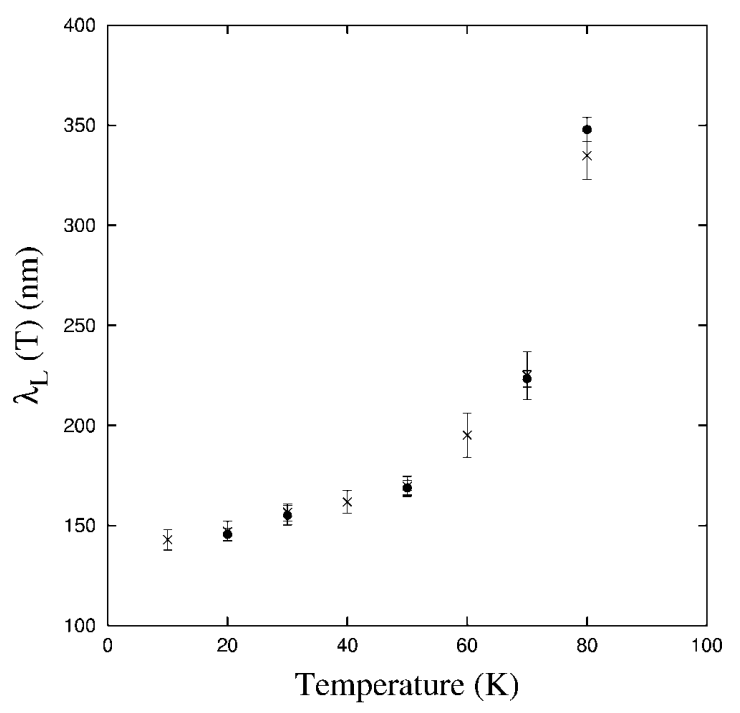

FIG. 4. The temperature dependence of the penetration depth $\lambda_{L}$ arising from supercurrents in the basal plane of our YBCO sample. (O) Values derived from the fits in Fig. 3. ( $\times$ ) Data from the field distributions observed in the mixed state of the same sample [25]. Excellent agreement is seen between the two techniques.

to Eq. (5), $z_{0}$ was allowed to be a free parameter. However, all the results were consistent with a temperatureindependent value of $8 \mathrm{~nm}$, so $z_{0}$ was set to this value in producing Fig. 3. The value of $z_{0}$ probably indicates, in addition to the effects of the $5 \mathrm{~nm}$ rms surface roughness, a unit cell or two of nonsuperconducting material. A similar result is noted in [23]. It is unlikely on both experimental and theoretical grounds that the nonlinear Meissner effect $[5,8]$ or nonlocal effects $[9,24]$ can be invoked as an alternative explanation of the reduced shielding near the surface, under the conditions of our experiment. The actual variation of field with depth in Fig. 3 is well fitted by the theoretical expression (5), with no adjustable parameters other than $z_{0}$ and the temperature-dependent penetration depth $\lambda_{L}$. The temperature dependence of $\lambda_{L}$ is shown in Fig. 4. We find a very reasonable value at $20 \mathrm{~K}$ of $146 \pm 3 \mathrm{~nm}$. The values derived from the earlier indirect, mixed state measurements on the same film $[3,25]$ agree well with the data presented here, and thereby give confidence in both techniques.

In conclusion, we have made the first direct, microscopic measurement of magnetic field values near the surface of a superconductor in the Meissner state, providing a direct confirmation of the London formula. It is interesting to note that until now textbook figures (for example [6]), which illustrate the penetration of a magnetic field into a superconductor in the Meissner state have had to rely on theoretical plots, since no direct experimental data have been available. A direct experimental measurement, even in a material where London theory is expected to apply, is therefore of archival importance, and also opens the possibility of investigating cases where Eq. (3) does not apply. These measurements demonstrate the general utility of the low energy muon technique for investigating samples over depths of order 20-150 nm with a few nm resolution. They also provide an indication of the reliability of simulations of the muon implantation process. Finally, we note that the unique possibilities offered by low energy muons are not restricted to studies of superconducting or magnetic thin films and multilayers, but may be extended to near surface studies of single crystals too.

This work was supported by the UK EPSRC, the German BMBF, and the Paul Scherrer Institute. The TRIM.SP source code was kindly provided by $\mathrm{W}$. Eckstein. We are grateful to H. P. Weber, D. George, and S. M. Brookes for technical support.

[1] E. Morenzoni et al. (to be published).

[2] V. Lauter-Pasyuk et al., Physica (Amsterdam) 267B\&268B, 149 (1999).

[3] Ch. Niedermayer et al., Phys. Rev. Lett. 83, 3932 (1999).

[4] W. N. Hardy et al., Phys. Rev. Lett. 70, 3999 (1993).

[5] J. E. Sonier et al., Phys. Rev. Lett. 83, 4156 (1999).

[6] J. Waldram, Superconductivity of Metals and Cuprates (Institute of Physics, Bristol, 1996).

[7] J. Halbritter, Z. Phys. 243, 201 (1971).

[8] S. K. Yip and J. Sauls, Phys. Rev. Lett. 69, 2264 (1992).

[9] M. H. S. Amin, I. Affleck, and M. Franz, Phys. Rev. B 58, 5848 (1998).

[10] C.P. Bidinosti, W. N. Hardy, D. A. Bonn, and Ruixing Liang, Phys. Rev. Lett. 83, 3277 (1999).

[11] S. T. Johnson et al., Phys. Rev. Lett. 82, 2792 (1999).

[12] J. Bonevich et al., Phys. Rev. B 57, 1200 (1998).

[13] K. A. Moler, J.R. Kirtley, D. G. Hinks, T. W. Li, and M. Xu, Science 279, 1193 (1998).

[14] M. W. Coffey, Phys. Rev. B 57, 11648 (1998).

[15] A. Mansour et al., Physica (Amsterdam) 156B\&157B, 867 (1989).

[16] E. Morenzoni et al., Hyperfine Interact 97\&98, 395 (1996).

[17] B. Utz, S. RiederZecha, and H. Kinder, IEEE Trans. Supercond. 7, 1272 (1997).

[18] B. D. Rainford and G. J. Daniell, Hyperfine Interact 87, 1129 (1994).

[19] T. M. Riseman and E. M. Forgan (to be published).

[20] W. Eckstein, Computer Simulations of Ion-Solid Interactions (Springer-Verlag, Berlin, 1992).

[21] H. Glückler et al. (to be published).

[22] A. Hofer, thesis, Universität Konstanz, 1998.

[23] V. Lauter-Pasyuk et al., Physica (Amsterdam) 248B, 166 (1998).

[24] I. Kosztin and A.J. Leggett, Phys. Rev. Lett. 79, 135 (1997).

[25] M. Pleines et al. (to be published). 